\title{
Correction to: PFKFB4 is overexpressed in clear-cell renal cell carcinoma promoting pentose phosphate pathway that mediates Sunitinib resistance
}

Chenchen Feng ${ }^{1,2+}$, Yuqing $\mathrm{Li}^{1,2+}$, Kunping $\mathrm{Li}^{1,2+}$, Yinfeng Lyu ${ }^{1,2+}$, Wenhui Zhu ${ }^{1,2}$, Haowen Jiang ${ }^{1,2^{*}}$ and Hui Wen ${ }^{1,2^{*}}$

\section{Correction to: J Exp Clin Cancer Res 40, 308 (2021)}

https://doi.org/10.1186/s13046-021-02103-5

Following publication of the original article [1], the authors identified some minor errors in Fig. 9, specifically:

- In Fig. 9C, the image of SRC-3 in KD group; the incorrect image was used

- In Fig. 9D, the image of tumor in Sun group; the incorrect image was used

The corrected figures are given here.

In addition, the Acknowledgements section has been corrected; the updated text is as follows:

\section{Acknowledgements}

The authors thank Dr Guoqing Ji for critical technical support.

The corrections do not have any effect on the final conclusions of the paper. The original article has been corrected.

\begin{abstract}
Author details
${ }^{1}$ Department of Urology, Huashan Hospital, Fudan University, 12 Middle Urumqi Rd, 200040 Shanghai, PR China. ${ }^{2}$ Institute of Urology, Fudan University, 200040 Shanghai, PR China.
\end{abstract}

Published online: 02 December 2021

\section{Reference}

1. Feng C, Li Y, Li K, et al. PFKFB4 is overexpressed in clear-cell renal cell carcinoma promoting pentose phosphate pathway that mediates Sunitinib resistance. J Exp Clin Cancer Res. 2021;40:308 https://doi.org/10.1186/ s13046-021-02103-5. 
A
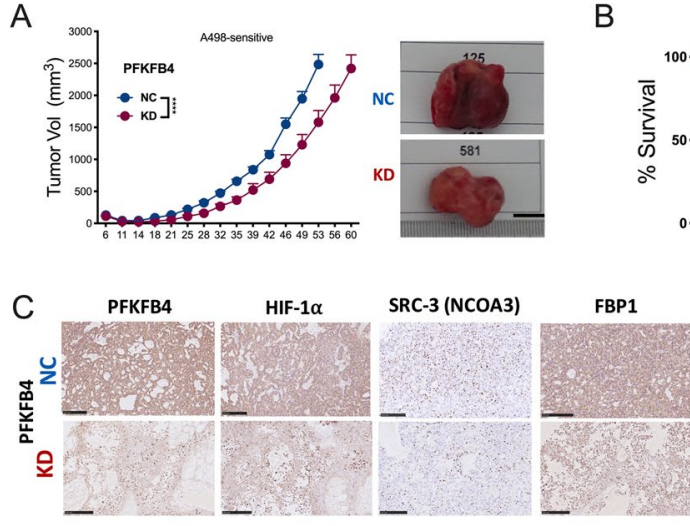

B
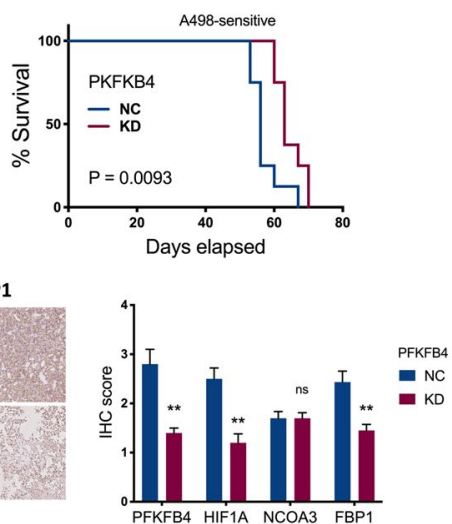

D

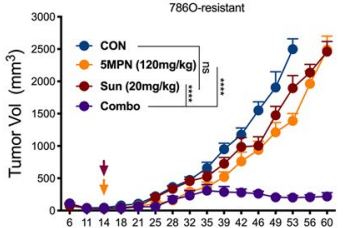

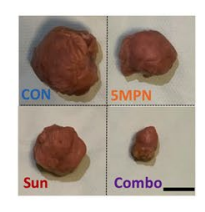

E

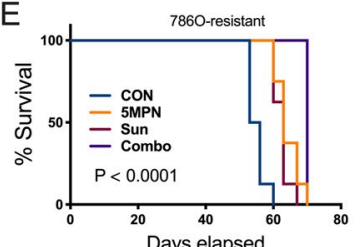

F
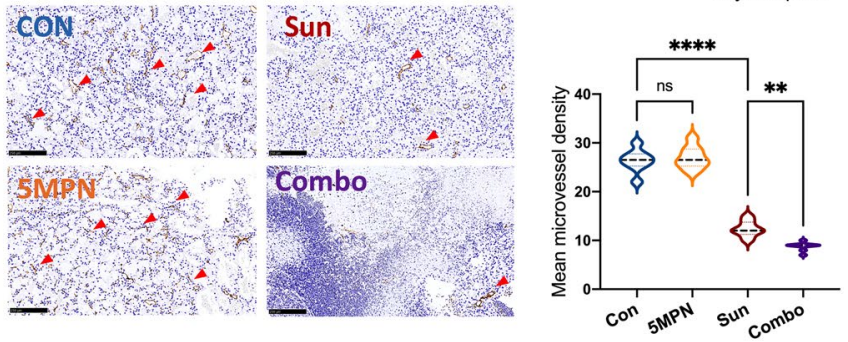

G
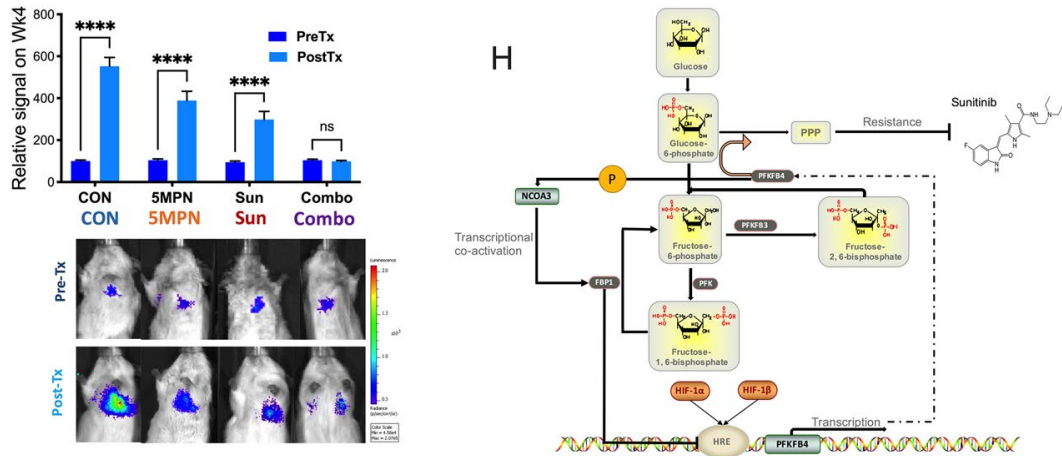

Fig. 9 PFKFB4-knockdown overcomes Sunitinib resistance in cCRCC in vivo. A Xenograft murine models consisting of 8 male BALB/c nude mice per group with subcutaneous implanted Sun-sensitive A498 cells with or without PFKFB4-KD (shRNA\#2) under right hind limb with tumor growth monitored over 60-day period and tumor size of $<2500 \mathrm{~mm} 3$ as endpoint, with representative tumor image at endpoint (bar $=1 \mathrm{~cm}$ ) analyzed by two-way ANOVA and B Kaplan-Meier curves of survival of mice, analyzed by Log-rank test; $\mathbf{C}$ Representative immunohistochemical staining of factors in tumors from A), with intensity scored semi-quantitatively and statistically compared; $\mathbf{D}$ Xenograft murine models consisting of 8 male BALB/c nude mice per group with subcutaneous implanted Sun-resistant 7860 cells fed with $20 \mathrm{mg} / \mathrm{kg}$ of Sun and/or $120 \mathrm{mg} / \mathrm{kg}$ of $5 \mathrm{MPN}$ orally by gavage; tumor growth monitored over 60-day period, analyzed by two-way ANOVA and $\mathbf{E}$ Kaplan-Meier curves of survival of mice, analyzed by Log-rank test; $\mathbf{F}$ Representative immunohistochemical staining CD31 targeting micro-vessels in tumors (red arrows) from $\mathbf{D}$, with relative micro-vessel density (MVD) statistically compared; G Tail vein injection of Sun-resistant A498 cells in 9 mice per group with 5MPN, Sun or combo treatments (Tx); mice monitored for 4 weeks for photon detection, normalized to PreTx CON group; bar figures showing photon change before (PreTx) and after (PostTx) with representative luciferase image showing lung involvement at endpoint of each group $\mathbf{H}$ Schematic cartoon of regulatory axis proposed by the current study. (All in vitro assays performed in triplicates and at least 3 biological replicates; ns $=$ not significant; ${ }^{*} P$ $<0.05$; ${ }^{* *} P<0.01$; ${ }^{* *} P<0.001$; ${ }^{* * *} P<0.0001$ ) 